\title{
Pattern of physician consultations from different departments of smhs and associated hospitals for the months of November 2011 and December 2011- medical council of India (mci) dercognition is not too far
}

\author{
${ }^{1}$ DR Shabir A Bhat, ${ }^{2} \mathrm{Dr}$ Abdul Majid Wani*, ${ }^{3} \mathrm{Dr}$. Mubeena Akhtar \\ Medical College Srinagar
}

Corresponding author's email: dr_wani_majid@yahoo.co.in

\begin{abstract}
The study was conducted to assess the pattern of inter hospital and inter-departmental consultations for in-patients and to ascertain whether the consultations made were desirable as per our setup in various hospitals of GMC Srinagar and avoid medico legal pitfalls and to conserve resources. All the consultations made from 10.0 am to $4.0 \mathrm{pm}$ from various departments and associated hospitals of GMC Srinagar were recorded and analyzed. The consultations were attended by the consultant in -charge medical casualty. The consultations made from Nov 2, 2011 to Dec 31, 2011 were included in the study. After the first month restraint was shown by a circular from the head of the department of medicine to various departments and hospitals to ensure involvement of senior staff whether a second opinion is desirable and avoid involvement of patient care-givers i.e., attendants in the consultation procedure. Obstetrics and gynecology department had $\mathbf{8 2} \%$ consultations of undesirable nature and less than $\mathbf{1 0 \%}$ where those with consultant involvement. This was followed by anaesthesia department and orthopedics with $\mathbf{7 8 \%}$ and $89 \%$ of the consultations respectively do undesirable nature. All this reflected junior staff involvement in patient care and high morbidity and mortality and shifting the medico legal responsibility to physician on call. Most of the consultations from departments of anaesthesia, obstetrics and gynecology and orthopedics where for shifting the responsibility on to physician and giving free hand to junior staff to manage sick patients without consulting their senior colleagues. This has an impact on patient outcome especially in obstetric patients and delay in surgical intervention in orthopedic and other elective surgeries in associated hospitals. Consultation policy is need of the hour to improve quality of health care in our setup.
\end{abstract}

Keywords: Physician, Desirable, Undesirable, Hospitals.

\section{INTRODUCTION}

Quality means optimizing material inputs and practitioner skill to produce health. These deficiencies in quality of care represent neither the failure of professional compassion nor necessarily a lack of resources (1). Rather, they result from gaps in knowledge, inappropriate applications of available technology (2), or the inability of organizations to change (3). Local health care systems may have failed to align practitioner incentives and objectives, to measure clinical practice, or to link quality improvement to better health outcomes. Quality health care is a dream to our population and it is not lack of resources and infrastructure but the lack of will to improve quality and lack of skill how to utilize available resources for diagnostic and therapeutic purposes.

Need of the hour is use of evidence based medicine in routine practice and put forth policies and protocols for critical care, infection control, diagnostic tests and treatment. 
Table 1. Showing pattern of consultations and referring departments

\begin{tabular}{|l|l|c|c|c|c|c|}
\hline 1 & Department & $\begin{array}{c}\text { Nov- } \\
\mathbf{1 1}\end{array}$ & $\begin{array}{c}\text { Dec- } \\
\mathbf{1 1}\end{array}$ & Desirable/indicated & $\begin{array}{c}\text { Not desirablel } \\
\text { Not indicated }\end{array}$ & $\begin{array}{c}\text { Referring Consultant } \\
\text { involved }\end{array}$ \\
\hline 2 & Surgical dept & 18 & 7 & 19 & 6 & 5 \\
\hline 3 & Siccu & 3 & 2 & 5 & 0 & 2 \\
\hline 4 & Ent & 3 & 3 & 4 & 2 & 1 \\
\hline 5 & Dermatology & 1 & 2 & 3 & 0 & 1 \\
\hline 6 & Opthalmology & 3 & 1 & 3 & 1 & 1 \\
\hline 7 & Dental & 2 & 1 & 3 & 0 & 3 \\
\hline 8 & Pyschiatry & 3 & 2 & 5 & 0 & 3 \\
\hline 9 & Chest disease & 3 & 2 & 2 & 1 & 2 \\
\hline 10 & Bone and joint hospital & 120 & 72 & 22 & 170 & 7 \\
\hline 11 & Maternity hospital-Lal ded & 134 & 61 & 43 & 152 & 9 \\
\hline 12 & Anaesthesia department & 106 & 112 & 44 & 174 & 1 \\
\hline
\end{tabular}

\section{METHODOLOGY}

This prospective study was conducted in Government Medical College (GMC) Srinagar Kashmir, India and associated hospitals with cumulative bed capacity of 3500.The hospitals have facilities of medicine and allied super specialties, surgery and allied super specialties, orthopedics, Obestetics and Gynecology, chest medicine and psychiatric medicine. All the consultations made from $10.0 \mathrm{am}$ to $4.0 \mathrm{pm}$ from various departments and associated hospitals of GMC Srinagar were recorded and analyzed.The consultations were attended by the consultant in -charge medical casualty. The consultations made from Nov 2, 2011 to Dec 31, 2011 were included in the study. After the first month restraint was shown by a circular from the head of the department of medicine to various departments and hospitals to ensure involvement of senior staff whether a second opinion is desirable and avoid involvement of patient care-givers i.e., attendants in the consultation procedure. The data was tabulated and analyzed by simple percentage methods.

\section{RESULTS}

Most of the consultations were made from maternity hospital, bone and joint hospital and department of anaesthesia. Department of anaesthesia made largest number of consultations out of which only $18 \%$ were desirable i.e. where physician opinion could be useful for patient care. Maternity hospital was the second to make physician consultations with only $22.05 \%$ desirable which could benefit the patient care. Bone and joint hospital made 192 physician consultations out of which only $11.45 \%$ (22) were desirable and helpful for patient care (Table 1). Following the circular from department of medicine there was $37.2 \%$ and $31.28 \%$ reduction in the number of consultations from bone and joint hospital and maternity hospital respectively. However, department of anaesthesia sent more consultations in the second month of December 112 as compared to 106 in preceding month (Graph 1).Involvement of consultant in-charge in consultation process was dismal with 1 consultation out of $212(0.47 \%)$ from anaesthesia, 7 out of 192(3.6\%) from bone and joint hospital and 9 out of 195 (4.6\%) from maternity hospital where consultant was involved somehow.

\section{DISCUSSION AND RECOMMENDATIONS}

Interdepartmental and inter hospital consultations are made all over the world for sake of patient care, teaching purposes, transfer of specialty care for which facilities are not available at that centre or department(1). Consultations act as opening channels of communication between departments -may increase the effectiveness of communication and decrease friction on the clinical services. in our setup most of the consultations were for clearance for surgery and opinion about X-ray chest and electrocardiograph (EKG). The need of the hour is to get all the $x$-rays reported and EKG interpreted by the concerned radiology department and anaesthetist. If such requests are for medico legal reasons then hospital has to adopt the policies and procedures for surgery and protocols for pre- and post-operative care. In the era of evidence based medicine we need to keep pace with rest of the world. We are liable to medico legal pitfalls and litigation in case we continue to act in vogue and ignore patient rights. Accreditation of departments and institutions need proper documentation, confidentiality of records, protocols and policies.

Consultations between medical and surgical residents occur frequently in both the inpatient and outpatient setting. Several reports have suggested that joint management of patients can reduce postoperative 
Figure 1. Showing graphic presentation of the data tabulated above.

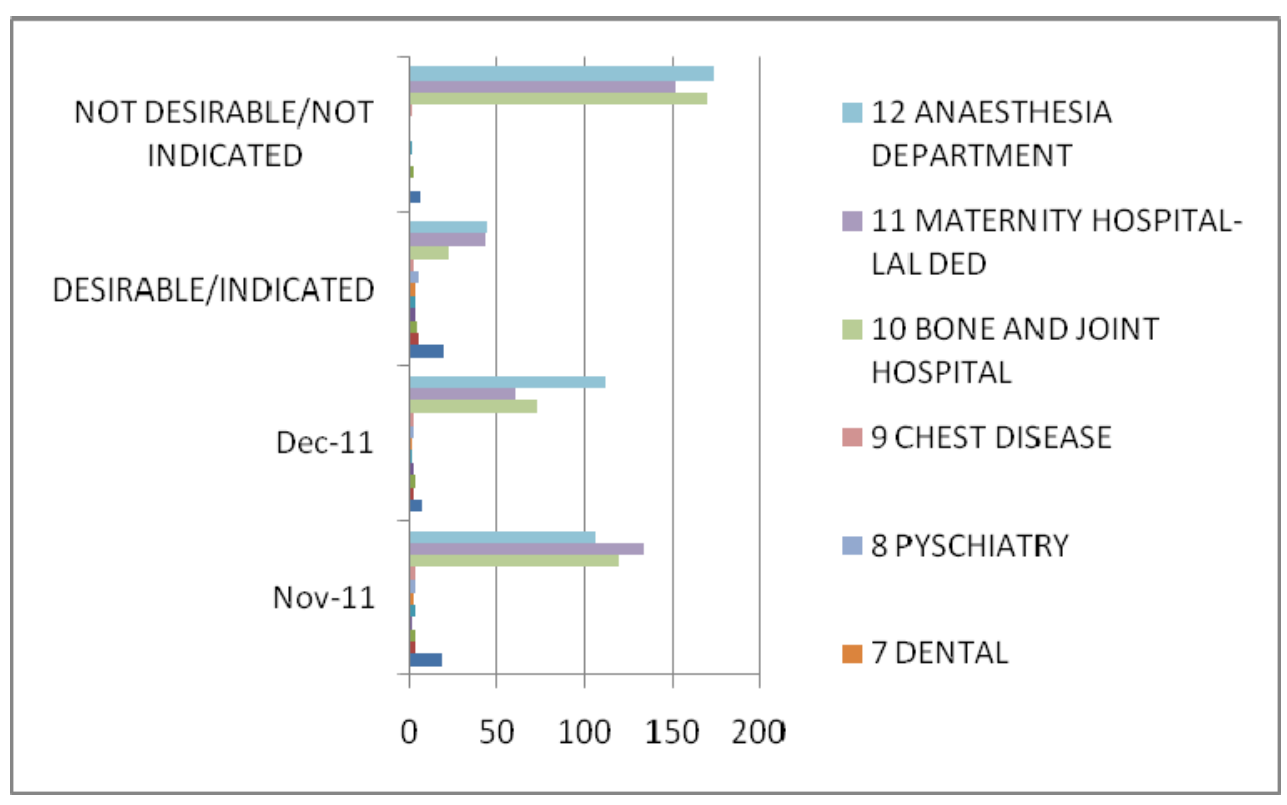

complications, increase adherence to treatment guidelines, and decrease preoperative length of stay $(2,3)$. We need to frame such guidelines of patient care as per international standards. Although there is little data regarding the adequacy of instruction in "consultation medicine" during residency, one graduate survey targeted communication strategies and management of common consultative problems as areas for of residents' interactions with other healthcare providers may be useful in order to best prepare graduates for practice and to comply with residency accreditation requirements (4).

As the Accreditation Council for postgraduate Medical Education shifts to outcomes-based accreditation standards, residency programs will need to provide education and monitor their trainees' achievements in core competencies such as professionalism, systemsbased practice, and interpersonal skills and communication (5). Our consultations lack theme, do not address the core issue, lack professionalism and do not involve senior staff like consultants to pinpoint the purpose of consultation. Using an intern as porter and a senior trainee as communicator without teaching them how to address the problem is un-ethical as same trainees are future consultants and physicians. We have created a vicious cycle of lack of accountability ignorance and get-going tactics which will deprive our future physicians and community from quality health care-the slogan all over the world and we will never be able to have accreditation with organizations like Joint Commission for Accreditation of Health Institutions (JCAHI). Thus, formal education in consultation medicine and evaluation of residents' interactions with other healthcare providers may be useful in order to best prepare graduates for practice and to comply with residency accreditation requirements. We need to have consultation policy, policies and procedures for surgery, protocols and guidelines for disease management as per evidence based medicine and international guidelines, change in teaching technique of our future doctors and create accountability to meet the needs of our ailing and ignorant patients for which all of us hold'"' Hippocratic Oath"'".

\section{ACKNOWLEDEGEMENTS}

We acknowledge the support and special grant from Cadila Pharmaceuticuals Ahmadabad, Gujrat India for publication of the manuscript and thus helping in improvement of health care in our setup.

\section{REFERENCES}

ACGME Outcome Project (2004). Accreditation Council for Graduate Medical Education Web site. Internet (http://www.acgme.org/ Outcome/). Retrieved September 8, 2004. Accessed September 8.

Berwick D (1989). Continuous Improvement as an Ideal in Health Care. New England Journal of Medicine, 320(1):53-56.

Devor M, Renvall M, Ramsdell J (1993). Practice patterns and the adequacy of residency training in consultation medicine. J Gen Intern Med; 10:554-60.

Huddleston JM, Long $\mathrm{KH}$, Naessens JM, Vanness D, Larson D, Trousdale R, Plevak (2004). Medical and surgical comanagement after elective hip and knee arthroplasty. Ann Intern Med.; 131:28-38. Institute of Medicine (2001). Crossing the Quality Chasm. Washington, DC: National Academy Press.

Keating NL, Landrum MB, Ayanian JZ, Winer EP, Guadagnoli E (2003). Consultation with a medical oncologist before surgery and type of 
surgery among elderly women with early-stage breast cancer. J Clin Oncol.; 21:4532-9.

Macpherson DS, Lofgren RP (1994). Outpatient internal medicine preoperative evaluation: a randomized clinical trial. Med Care, 32:498-507.
Murray CJ, Frenk JA (2000). Framework for Assessing the Performance of Health Systems. Bulletin of the World Health Organization, 78(6):717-31. 